\title{
Utility of magnetic resonance spectroscopy and diffusion-weighted imaging for detecting changes in the femoral head in divers with hip pain at risk for dysbaric osteonecrosis
}

\author{
Tsung-Tai Lin ${ }^{1}$, Cheng-Chuan $\mathrm{Hu}^{1}$, Yi-Chih Hsu ${ }^{1}$, Chih-Chien Wang ${ }^{2}$, Shih-Wei Chiang ${ }^{1}$, \\ Chao-Ying Wang ${ }^{3}$, Wei-Chou Chang', Guo-Shu Huang ${ }^{1,4}$ \\ ${ }^{1}$ Department of Radiology, Tri-Service General Hospital, National Defense Medical Center, Taipei, Taiwan; ${ }^{2}$ Department of Orthopedic Surgery, \\ Tri-Service General Hospital, National Defense Medical Center, Taipei, Taiwan; ${ }^{3}$ Department and Graduate Institute of Biology and Anatomy, \\ National Defense Medical Center, Taipei, Taiwan; ${ }^{4}$ Department of Medical Research, Tri-Service General Hospital, National Defense Medical \\ Center, Taipei, Taiwan
}

Contributions: (I) Conception and design: YC Hsu, GS Huang; (II) Administrative support: TT Lin, CC Hu; (III) Provision of study materials or patients: CC Wang; (IV) Collection and assembly of data: TT Lin, CC Hu, YC Hsu, SW Chiang, CC Wang, GS Huang; (V) Data analysis and interpretation: TT Lin, CC Hu, YC Hsu, SW Chiang, CY Wang, WC Chang, GS Huang; (VI) Manuscript writing: All authors; (VII) Final approval of manuscript: All authors.

Correspondence to: Yi-Chih Hsu, MD; Guo-Shu Huang, MD. Department of Radiology, Tri-Service General Hospital, 325, Cheng-Kung Road, Sec. 2, Taipei 114202, Taiwan. Email: doc31578@gmail.com; gshuang5@gmail.com.

Background: Ischemia before the development of dysbaric osteonecrosis (DON) in femoral heads has never been investigated. We assessed whether quantitative magnetic resonance spectroscopy (MRS) and diffusion weighted imaging (DWI) could detect dysbaric changes in divers with hip pain.

Methods: This IRB-approved exploratory study recruited 17 divers [9 with hip pain (Group 1); 8 asymptomatic (Group 2)] with normal findings on radiographs and conventional magnetic resonance imaging scans were age-, gender- and body-mass-index matched to 17 non-divers as controls (Group 1C, 2C). Apparent diffusion coefficients (ADCs) and MRS spectra were obtained from regions/voxels of interest on the femoral heads of all subjects. LCModel was used to determine water content, lipid composition, and the unsaturation index in bone marrow. Mann-Whitney non-parametric test was used to compare results of quantitative MRS and ADCs of ipsilateral femoral heads between divers and controls.

Results: MRS of the ipsilateral femoral heads revealed higher water (peak: $4.7 \mathrm{ppm}$ ) content, lower total lipid fraction (TLF), and higher unsaturation index (UI) of lipids in Group 1 than in Group 2 (water: $\mathrm{P}=0.040$; UI: $\mathrm{P}=0.022$ ) and Group 1C (water: $\mathrm{P}=0.027$; TLF: $\mathrm{P}=0.039$; UI: $\mathrm{P}=0.009$ ). In contrast, femoral head ADCs were comparable between divers and controls. Five out of nine symptomatic divers were contacted for follow-up MRS and DWI studies, and the mean difference in water content in the femoral heads of patients with osteonecrosis was also higher than that in patients with symptom relief (osteonecrosis: $0.077 \pm 0.130$ vs. symptom relief: $0.003 \pm 0.010$ ).

Conclusions: Dysbaric change in the femoral heads of divers with hip pain can be detected using quantitative MRS, which reveals increases in water content and UI of lipids, and a decrease in TLF.

Keywords: Dysbaric change; diving; femoral head; magnetic resonance spectroscopy (MRS); diffusion-weighted magnetic resonance imaging (diffusion-weighted MRI)

Submitted Feb 05, 2021. Accepted for publication Jun 02, 2021.

doi: $10.21037 /$ qims-21-148

View this article at: https://dx.doi.org/10.21037/qims-21-148 


\section{Introduction}

Dysbaric osteonecrosis (DON) is an occupational hazard among divers and compressed air workers, and characteristically occurs in long bones containing fatty marrow such as the humerus, femur, and tibia (1). It mostly results from rapid decompression after prolonged hyperbaric exposure, which causes intravascular nitrogen gas bubbles to form and aggregate and give rise to nitrogen emboli and local ischaemia in the bone marrow. In addition, the nitrogen gas bubbles formed in bone marrow or fat cells may cause compartment syndrome or fat cell rupture followed by fat embolism (2-4). According to previous studies, DON occurs commonly in the head of the femur and humerus. It is mostly asymptomatic at the initial ischaemic stage, but juxta-articular lesions in the hips and shoulders are most likely to become symptomatic even when they have a normal appearance on plain radiographs or conventional sequences of magnetic resonance imaging (MRI) $(1,5)$.

Previous studies have used magnetic resonance spectroscopy (MRS) and diffusion-weighted imaging (DWI) to assess the initial ischaemic stage of osteonecrosis in femoral heads (6-8). Hou et al. reported that proton MRS could depict alterations in the lipid-water compositions of normal-appearing femoral heads with and without nontraumatic and non-steroid induced osteonecrosis in the contralateral hip (8). In addition, Menezes et al. observed that the diffusion process became sensitive to early ischaemia in piglets' legs when the supplying arteries were ligated (7). The apparent diffusion coefficient (ADC) decreases in a few hours owing to the decrease in blood perfusion. Epiphyseal osteonecrosis results in an increase in the ADC when collateral circulation is formed. Hip pain after diving, which may be associated with dysbaric ischemia, has never before been evaluated using MRS and DWI.

In this case-control study, we used non-contrast MRI to detect dysbaric change before the development of DON in the femoral heads of divers. As hip pain was proved to be associated with elevating intraosseous pressure in patients with femoral head osteonecrosis (9), we supposed that hip pain in divers was related to dysbaric change in the femoral head, even though the femoral head's appearance is normal on plain radiographs and conventional sequences of MRI. This study aimed to investigate whether quantitative MRS and DWI can be used to detect dysbaric change before osteonecrosis development, and thus prevent DON at an early stage before detection by conventional imaging.

\section{Methods}

\section{Subjects}

The study was conducted in accordance with the Declaration of Helsinki (as revised in 2013). This study was approved by the Institutional Review Board of our hospital. All participants provided written informed consent to undergo plain radiographs and non-contrast MR studies, including MRS and DWI, of bilateral hips during the period of 01 January 2014 to 31 December 2014. All imaging studies were performed once for each subject and were interpreted by two experienced musculoskeletal radiologists who confirmed that all enrolled subjects presented negative findings on plain radiographs and conventional MR studies. Before imaging studies, clinical assessment was performed by a single orthopaedic surgeon who recorded hip pain in divers. All enrolled divers denied previous history of long-term steroid use, alcohol use disorders (defined in the Diagnostic and Statistical Manual of Mental Disorders-V) (10), hip trauma, surgery, chronic hip pain, or lower back pain.

As the bone composition is associated with sex, age, and body-mass index (BMI) $(11,12)$, water content and lipid compositions in bone marrow might change with these parameters. Thus, the anthropometric data (sex, age, and BMI defined as body weight in kilograms divided by squared height in meters) of divers were collected to recruit gender-, age- and BMI-matched non-divers as controls. The results of quantitative MRS and DWI in the controls served as baseline data for the divers prior to diving.

We divided enrolled divers into two groups based on presence of hip pain, which was the most common initial symptom in patients with osteonecrosis according to a previous multicentre analysis (13). In total, we enrolled 17 occupational divers, among whom three had bilateral hip pain, six had unilateral hip pain, and eight were asymptomatic. We also selected as controls 17 nondivers who were gender-, age-, and BMI-matched to each diver. To confirm that dysbaric changes were reversible in symptomatic femoral heads with normal findings on plain radiographs and conventional MRI, we followed-up these symptomatic divers for 5 years to determine whether osteonecrosis occurred when hip pain persisted, and vice versa. Five out of nine symptomatic divers were contacted after 5 years to arrange for follow-up MRS and DWI studies with the same protocol. 

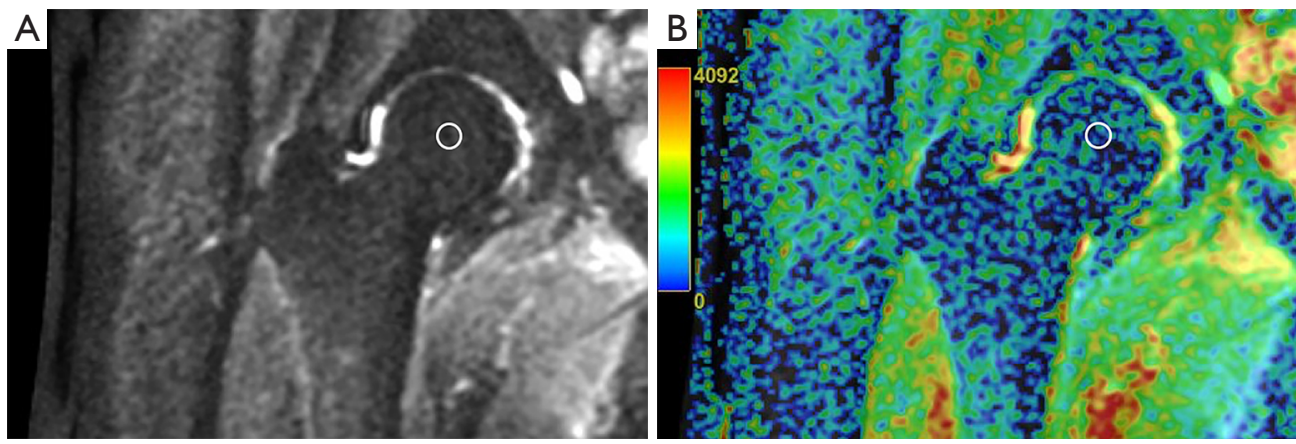

Figure 1 FOCUS-DWI and ADC maps of the femoral head in the midcoronal plane. (A) ADC is measured by positioning the ROI box in the femoral head ( $b=0$ and $400 \mathrm{~s} / \mathrm{mm}^{2}$ ) on DWI. (B) ADC maps are generated at the workstation after placing the box in the femoral head. ADC, apparent diffusion coefficient; DWI, diffusion-weighted imaging.

\section{MRI and DWI protocol}

All the studies were performed using the 3.0 Tesla (T) wholebody imaging system (Discovery MR750, GE Healthcare, Waukesha, WI). To assess the condition of the femoral head, the imaging protocol included a coronal T1-weighted fast spin-echo sequence [repetition time (TR)/echo time (TE) $=819 / \mathrm{min}$ full $(\mathrm{ms})$, field of view $(\mathrm{FOV})=40 \mathrm{~cm}]$ with a $4-\mathrm{mm}$ section thickness and 0.5 -mm section gap. Further, T2-weighted images (TR/TE $=2,354 / 125 \mathrm{~ms}, \mathrm{FOV}=40 \mathrm{~cm} \times 40 \mathrm{~cm})$ and T2-weighted images with fat saturation $(\mathrm{T} 2 \mathrm{FS}$; TR $/ \mathrm{TE}=3,063 / 68 \mathrm{~ms}, \mathrm{FOV}=40$ $\mathrm{cm} \times 40 \mathrm{~cm}$ ) were acquired in the coronal orientation with the same section thickness and gap. These conventional MR images were used to select the patients and individuals without any disease or femoral head abnormality, such as osteonecrosis, with or without deformity, infection, or neoplasm. DWI and proton MRS were then performed in the femoral heads with normal signal intensity and contours.

The ADC was measured using FOV optimised and constrained undistorted single-shot (FOCUS) DWI. FOCUS-DWI was used in this study because of its superiority to conventional DWI in a number of respects such as lesion conspicuity, image artefacts, image blurring, distortion, and overall image quality (14). FOCUSDWI was used to measure diffusion in six directions with $4,000 /$ minimum, $b=0$ and $400 \mathrm{~s} / \mathrm{mm}^{2}$ [number of excitations $(\mathrm{NEX})=20]$, 6-mm section thickness, and 16-cm FOV in an oblique coronal view. ADC maps were automatically generated by the scanner software. Mean ADC was measured with region of interest (ROI) technique on GE workstations off-line, using one circular ROI placed at the center of the femoral head in the midcoronal plane. The mean ADC values were obtained by averaging the values from four repeat ROIs of one single image (Figure 1). The size of the circular ROIs was chosen to be approximately $8 \mathrm{~mm}$ in diameter, and the mean ROI area and standard deviation were $50.2 \pm 10.1 \mathrm{~mm}^{2}$. All mean ADCs in femoral heads (proximal epiphysis) of divers and controls were measured by an attending radiologist with four years of experience in musculoskeletal diffusion-weighted MRI, who was blinded to the clinical data.

\section{MRS protocol and quantification}

MRS acquisition was performed in femoral heads using point-resolved spectroscopy (PRESS) for voxel localization. An automatic global shimming procedure was performed before each acquisition. MRS acquisition parameters were TR of 1,500 ms, TE of $35 \mathrm{~ms}$ to minimize T2 weighting, a cubic sample volume with dimensions $12.5 \times 12.5 \times 12.5 \mathrm{~mm}^{3}$, acquisition of 64 signals for each of the 4,096 data points without water suppression, and a spectral bandwidth of $5,000 \mathrm{~Hz}$. Each voxel was positioned by the same investigator in the femoral heads (proximal epiphysis) of all divers and non-diver controls (Figure 2A,2B). The size of the voxel of interest $(\mathrm{VOI})$ was set at $10 \times 10 \times 10 \mathrm{~mm}^{3}$.

Acquired proton MRS spectra showed a dominant peak at $1.30 \mathrm{ppm}$ and a series of lipid peaks at $3.0 \mathrm{~T}$ (Figure $2 B$ ). Each signal peak represented an individual functional group of lipids at a particular magnetic field value (Table 1) (15). In this study, functional groups of lipids were respectively detected at $0.90,1.30,1.59,2.03,2.25,2.77,4.10-4.35$, 5.2 , and $5.3 \mathrm{ppm}$, at $3.0 \mathrm{~T}$. The water peak was at $4.7 \mathrm{ppm}$ at 3.0 T. Raw data of spectra acquired from each diver and control were exported and analysed on an offline computer 

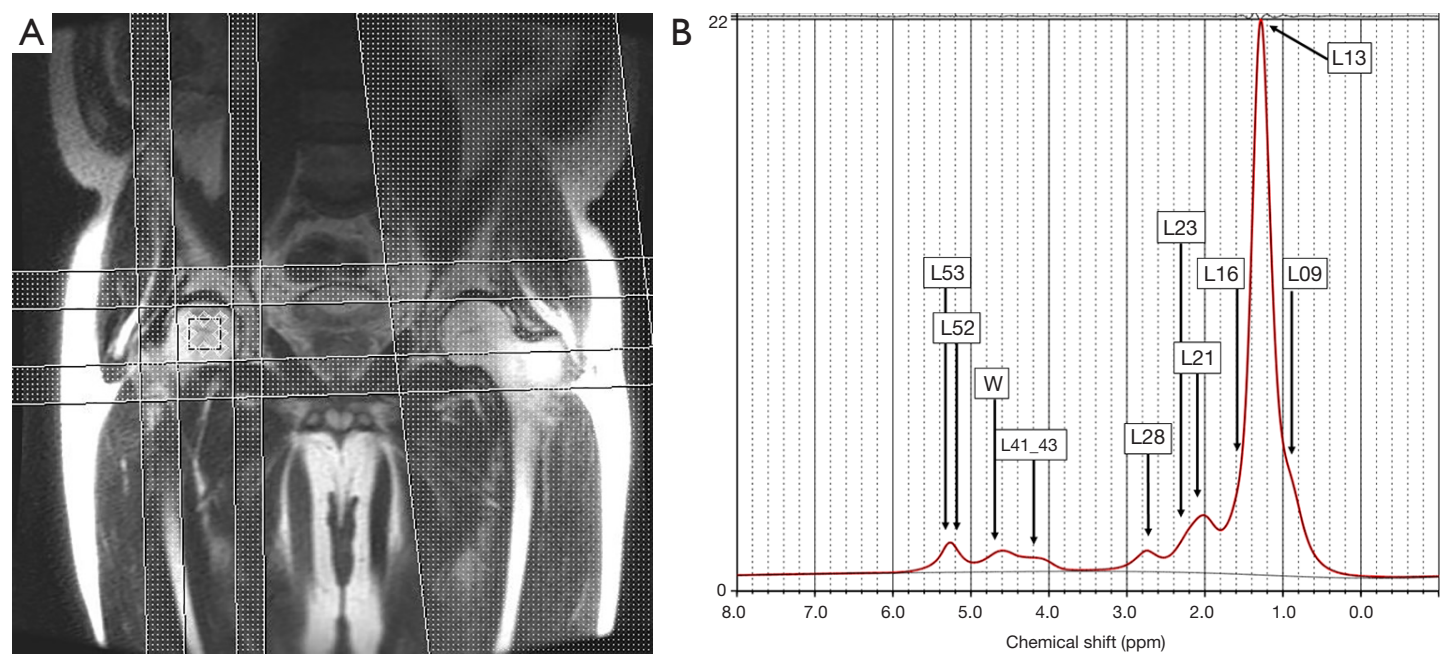

Figure 2 Point-resolved spectroscopy (PRESS) acquisition at 3.0 T from the femoral head. (A) The VOI box is placed in the femoral head of subjects for quantitative MRS analyses. (B) The LCModel software analyzes specific chemical shifts (arrows, also listed in Table 1) in the spectrum. MRS, magnetic resonance spectroscopy.

Table $1{ }^{1} \mathrm{H}$ NMR spectral peak assignments analyzed in this study (3.0 T magnetic field)

\begin{tabular}{|c|c|c|c|c|}
\hline Functional group & $\begin{array}{l}\text { Chemical shift ranges }{ }^{\dagger} \\
(\mathrm{ppm})\end{array}$ & Assignment & $\begin{array}{l}\text { Chemical shift for analysis in LCModel } \\
\qquad(\mathrm{ppm})\end{array}$ & Abbreviation \\
\hline Olefinic protons & $5.29-5.43$ & $-\mathrm{CH}=\mathrm{CH}-$ & 5.3 & L53 \\
\hline Water & 4.70 & $\mathrm{H}_{2} \mathrm{O}$ & 4.7 & W \\
\hline$\beta$-glycerol (methylene protons) & $4.10-4.35$ & $-\mathrm{CH}_{2} \mathrm{OCOR}$ & $4.10-4.35$ & L41, L43 \\
\hline$\alpha$-methylene protons & $2.25-2.36$ & $-\mathrm{OCO}-\mathrm{CH}_{2}-$ & 2.25 & L23 \\
\hline Allyl methylene protons & $1.93-2.13$ & $-\mathrm{CH}_{2}-\mathrm{CH}=\mathrm{CH}-$ & 2.03 & L21 \\
\hline$\beta$-methylene protons (carboxyl) & $1.55-1.69$ & $-\mathrm{OCO}-\mathrm{CH}_{2}-\mathrm{CH}_{2}-$ & 1.59 & L16 \\
\hline
\end{tabular}

${ }^{\dagger}$, the shown chemical shift ranges are previously published data (18-20) and these values are used as integration limits for measurement of peak areas.

using the LCModel software (Version 6.3-1L; Copyrighted @1992-2015 by Stephen Provencher), which had previously been applied for analysing the water and lipid composition of bone marrow $(16,17)$. Absolute concentrations of water and individual functional groups of lipids were obtained as described in the Table 1. In this analysis, one set of MRS data (water content) from a patient in group 1 and another set of MRS data (water content) from a patient in group 2 were discarded because the $\%$ SD was higher than $10 \%$.

Total lipid fraction (TLF) in bone marrow was calculated using the equation:

$$
T L F \%=\left[\Sigma I_{\text {lipid }} /\left(\Sigma I_{\text {lipid }}+I_{w}\right)\right] \times 100 \%
$$

where $\Sigma I_{\text {lipid }}$ was the sum of absolute concentrations of all functional groups of the lipids and $I_{w}$ was the absolute concentration of water. 
Table 2 Overview of anthropometric data in all subjects $(\mathrm{n}=34)$

\begin{tabular}{lccccc}
\hline Anthropometric data & Group 1 $(\mathrm{n}=9)$ & Group 1C $(\mathrm{n}=9)$ & P value & Group 2 $(\mathrm{n}=8)$ & Group 2C $(\mathrm{n}=8)$ \\
\hline Age (years) & $44.3 \pm 7.6$ & $44.1 \pm 8.4$ & 0.889 & $46.0 \pm 10.5$ & $49.4 \pm 9.9$ \\
BMI $\left(\mathrm{kg} / \mathrm{m}^{2}\right)$ & $25.1 \pm 4.8$ & $23.8 \pm 3.2$ & 0.524 & $25.2 \pm 3.2$ & $25.3 \pm 3.3$ \\
Gender (female/male) & $2 / 6$ & $2 / 6$ & $1 / 7$ & $1 / 7$ & 0.785 \\
\hline
\end{tabular}

Group $1(n=9)$ : symptomatic diver group. Group $2(n=8)$ : asymptomatic diver group. Group 1C ( $n=9)$ : matched control for group 1. Group $2 \mathrm{C}(\mathrm{n}=8)$ : matched control for group 2. All values are expressed as mean \pm standard deviation (SD). $P$ values of the student independent $t$-tests are demonstrated.

Table 3 Overview of diving exposure in all subjects $(\mathrm{n}=34)$

\begin{tabular}{lccc}
\hline Diving exposure data & Group 1 & Group 2 & P value \\
\hline Period (years) & $16.6 \pm 8.2$ & $17.4 \pm 9.1$ & 0.441 \\
Period (days/year) & $98.3 \pm 56.5$ & $163.3 \pm 88.0$ & 0.121 \\
Max. depth $(\mathrm{m})$ & $42.7 \pm 9.8$ & $44.7 \pm 11.4$ & 0.435 \\
\hline
\end{tabular}

Group $1(n=9)$ : symptomatic diver group. Group $2(n=8)$ : asymptomatic diver group. Group 1C ( $n=9)$ : matched control for group 1. Group $2 \mathrm{C}(\mathrm{n}=8)$ : matched control for group 2. All values are expressed as mean \pm standard deviation (SD). $P$ values of the student independent t-tests are demonstrated.

Marrow lipid unsaturation index (UI) was estimated using the equation:

$$
U I=I_{\text {Double }} /\left(I_{\text {Methyl }}+I_{\text {Methylene }}+I_{\text {Olefinic }}\right)
$$

where I Double was the absolute concentration of functional groups with double bonds (L21, L28, L53), and $I_{\text {Methyl }}, I_{\text {Methylene }}$, and $I_{\text {Olefinic }}$ were absolute concentrations of the methyl group (L09), methylene groups (L13, L16, L21, L23, L28, L41, L43 and L52), and olefinic group (L53), respectively.

\section{Statistical analysis}

In this study, we assigned symptomatic divers to Group $1(\mathrm{n}=9)$, asymptomatic divers to Group $2(\mathrm{n}=8)$, and their respective controls to Group $1 \mathrm{C}(\mathrm{n}=9)$ and Group $2 \mathrm{C}(\mathrm{n}=8)$. Student independent $t$-tests were used for comparing the mean ages and BMIs between the diver and control groups (i.e., Group 1 vs. Group 1C, Group 2 vs. Group 2C) and comparing the diving period and maximal diving depths between Groups 1 and 2. We used the Mann-Whitney non-parametric test to compare ADCs, water content, lipid compositions (i.e., TLF and absolute concentrations of functional groups of lipids), and UI in the bone marrows of ipsilateral femoral heads between the diver and control groups. All statistics were performed using SPSS software (IBM, version 22.0). Differences were considered statistically significant when the two-tailed $\mathrm{P}$ value was below 0.05 .

\section{Results}

Nine divers with unilateral or bilateral hip pain were included in Group 1 and eight asymptomatic divers were included in Group 2. The recruited divers reported their average diving experience as $17.1 \pm 8.5$ years (126.7 \pm 76.8 days/year) and average diving depth as $43.8 \pm 10.5$ metres. Decompression devices were used routinely by most occupational divers (16/17). The anthropometric data of the divers and controls and diving experience of Group 1 and Group 2 are listed in Tables 2,3. The ages and BMIs of both diver groups were comparable to their matched controls. There was no significant difference in diving period and maximal diving depth between Groups 1 and 2.

Mean ADCs, water content, lipid compositions, and UI of lipids in the femoral heads of divers and controls were presented in Table 4. The LCModel of the ipsilateral femoral head revealed higher water content in Group 1 than in Group 1C [Group 1: 8.49 $\pm 4.05\left(\times 10^{-2} \mathrm{mM}\right)$ vs. Group 1C: $\left.5.23 \pm 2.61\left(\times 10^{-2} \mathrm{mM}\right), \mathrm{P}=0.027\right]$, whereas no significant difference in water content was noted between Group 2 and Group 2C [Group 2: 5.81 $\pm 2.51\left(\times 10^{-2} \mathrm{mM}\right)$ vs. Group 2C: $6.85 \pm 5.24\left(\times 10^{-2} \mathrm{mM}\right), \mathrm{P}=0.983$ ] (Table 4). Besides, water 
Table 4 Mean ADCs, water content, lipid compositions and UI of lipids in femoral heads of divers and controls

\begin{tabular}{|c|c|c|c|c|c|c|c|}
\hline Parameters & \multicolumn{3}{|c|}{ Divers $(n=17)$} & \multicolumn{3}{|c|}{ Non-diver controls $(n=17)$} & $P$ value \\
\hline \multicolumn{8}{|l|}{ Mean ADC } \\
\hline \multirow[t]{2}{*}{$\operatorname{ADC}\left(\times 10^{-4}\right)$} & Group 1 & 4.77 & 1.35 & Group 1C & 4.42 & 0.89 & 0.389 \\
\hline & Group 2 & 4.60 & 0.75 & Group 2C & 4.40 & 0.89 & 0.633 \\
\hline \multirow[t]{2}{*}{$\mathrm{W}\left(\times 10^{-2} \mathrm{mM}\right)$} & Group 1 & 8.49 & 4.05 & Group 1C & 5.23 & 2.61 & 0.027 * \\
\hline & Group 2 & 5.81 & 2.51 & Group 2C & 6.85 & 5.24 & 0.983 \\
\hline \multicolumn{8}{|c|}{ Lipid compositions, TFC and UI (derived from LCModel) } \\
\hline $\log \left(\times 10^{-2} \mathrm{mM}\right)$ & Group 1 & 5.42 & 3.83 & Group 1C & 4.51 & 2.98 & 0.499 \\
\hline $\mathrm{L} 13\left(\times 10^{-2} \mathrm{mM}\right)$ & Group 2 & 7.11 & 2.02 & Group 2C & 7.28 & 0.92 & 0.263 \\
\hline \multirow[t]{2}{*}{$\mathrm{L} 16\left(\times 10^{-2} \mathrm{mM}\right)$} & Group 1 & 1.49 & 0.06 & Group $1 \mathrm{C}$ & 2.13 & 2.12 & 1.000 \\
\hline & Group 2 & 2.04 & 0.07 & Group 2C & 3.63 & 2.93 & 0.094 \\
\hline \multirow[t]{2}{*}{$\mathrm{L} 21\left(\times 10^{-2} \mathrm{mM}\right)$} & Group 1 & 5.87 & 2.75 & Group 1C & 4.03 & 1.84 & 0.109 \\
\hline & Group 2 & 4.44 & 2.60 & Group 2C & 5.17 & 1.51 & 0.232 \\
\hline \multirow[t]{2}{*}{$\mathrm{L} 23\left(\times 10^{-2} \mathrm{mM}\right)$} & Group 1 & 3.65 & 1.26 & Group 1C & 3.53 & 0.88 & 0.460 \\
\hline & Group 2 & 3.44 & 0.83 & Group 2C & 3.10 & 0.55 & 0.178 \\
\hline $\mathrm{L} 28\left(\times 10^{-2} \mathrm{mM}\right)$ & Group 1 & 1.83 & 0.45 & Group 1C & 1.54 & 0.60 & 0.117 \\
\hline \multirow[t]{2}{*}{$\mathrm{L} 52\left(\times 10^{-2} \mathrm{mM}\right)$} & Group 1 & 1.30 & 1.24 & Group $1 \mathrm{C}$ & 1.49 & 1.09 & 0.460 \\
\hline & Group 2 & 0.71 & 0.69 & Group 2C & 1.04 & 1.03 & 0.494 \\
\hline \multirow[t]{2}{*}{$\mathrm{L} 53\left(\times 10^{-2} \mathrm{mM}\right)$} & Group 1 & 2.89 & 1.16 & Group 1C & 2.79 & 1.28 & 0.976 \\
\hline & Group 2 & 3.20 & 1.46 & Group 2C & 3.42 & 1.24 & 0.407 \\
\hline \multirow[t]{2}{*}{ TLF (\%) } & Group 1 & 86.8 & 17.4 & Group 1C & 94.9 & 2.3 & $0.039^{\star}$ \\
\hline & Group 2 & 94.3 & 1.8 & Group 2C & 93.3 & 4.9 & 0.724 \\
\hline \multirow[t]{2}{*}{ UI $\left(\times 10^{-1}\right)$} & Group 1 & 1.23 & 0.42 & Group $1 \mathrm{C}$ & 0.85 & 0.27 & $0.009^{*}$ \\
\hline & Group 2 & 0.92 & 0.33 & Group 2C & 1.05 & 0.14 & 0.783 \\
\hline
\end{tabular}

One set of MRS data in Group 2 is lost in this study. ${ }^{*}$, statistical significance of Mann-Whitney non-parametric test. TLF, total lipid fraction. $\mathrm{mM}: \mathrm{mmol} / \mathrm{kg}$. Ul, unsaturation index. 

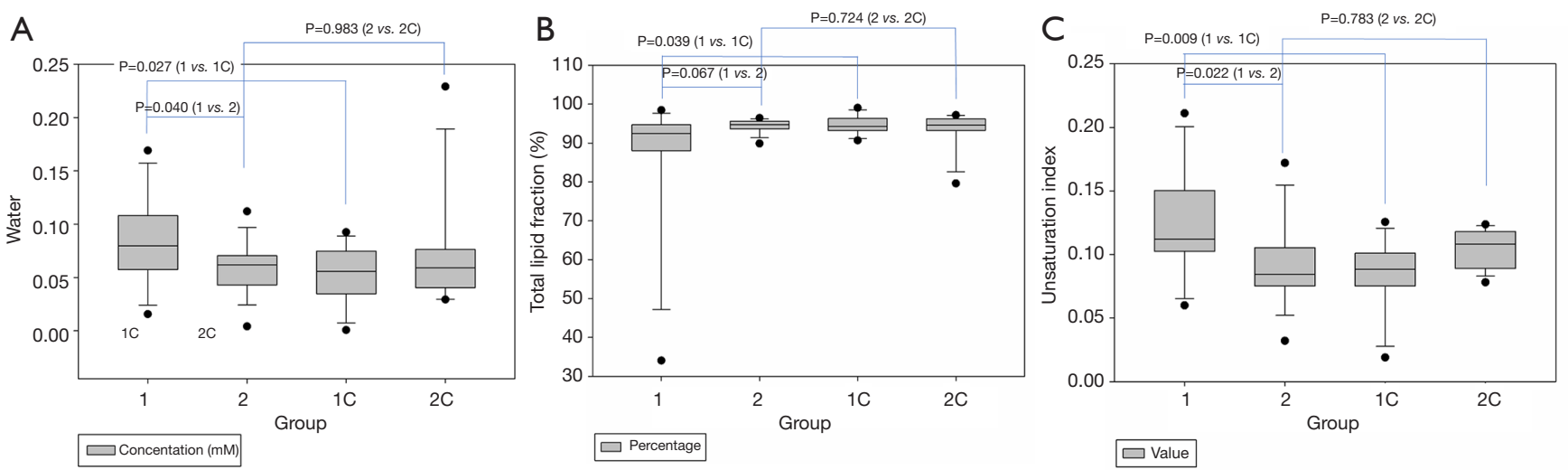

Figure 3 MRS of the ipsilateral femoral heads revealed (A) higher water content, (B) lower total lipid fraction and (C) higher unsaturation index of lipids in Group 1 than in Group 2 and Group 1C (i.e., the control group of Group 1).

content in the femoral heads of Group 1 was also higher than in Group 2 [Group 1: 8.49 $\pm 4.05\left(\times 10^{-2} \mathrm{mM}\right)$ vs. Group 2: $\left.5.81 \pm 2.51\left(\times 10^{-2}\right), \mathrm{P}=0.040\right)$ (Figure $3 A$ ). Comparison of water content between the two bilateral femoral heads of divers with unilateral hip pain revealed the presence of more intramedullary water on the painful side than on the nonpainful side; however, no statistical significance was noted [painful side: $7.49 \pm 3.85\left(\times 10^{-2} \mathrm{mM}\right) v s$. nonpainful side: $\left.5.37 \pm 2.84\left(\times 10^{-2} \mathrm{mM}\right), \mathrm{P}=0.325\right]$. In addition, water concentration was comparable between pain-free femoral heads of unilaterally symptomatic divers and ipsilateral femoral heads of controls [unilaterally symptomatic divers: $5.37 \pm 2.88\left(\times 10^{-2}\right) v s$. controls: $4.39 \pm 2.52\left(\times 10^{-2} \mathrm{mM}\right)$, $\mathrm{P}=0.529]$.

Even though there was no significant difference in the concentrations of functional groups of lipids, the total lipid fraction (TLF) in femoral heads was significantly lower in Group 1 than Group 1C (Group 1: $86.8 \% \pm 17.4 \%$ vs. Group 1C: $94.9 \% \pm 2.3 \%, \mathrm{P}=0.039)$, but comparable in Group 2 and Group 2C (Group 2: 94.3\% $\pm 1.8 \%$ vs. Group 2C: $93.3 \% \pm 4.9 \%, \mathrm{P}=0.724$ ) (Figure 3B). Moreover, the UI of lipids in femoral heads was significantly higher in Group 1 than Group 1C [Group 1: $1.23 \pm 0.42\left(\times 10^{-1}\right)$ vs. Group 1C: $0.85 \pm 0.27\left(\times 10^{-1}\right), \mathrm{P}=0.009$ ] and Group 2 [Group 1: $1.23 \pm 0.42\left(\times 10^{-1}\right) v s$. Group 2: $\left.0.92 \pm 0.33\left(\times 10^{-1}\right), \mathrm{P}=0.022\right]$, but comparable between Group 2 and Group 2C [Group 2: $0.92 \pm 0.33\left(\times 10^{-1}\right)$ vs. Group 2C: $\left.1.05 \pm 0.14\left(\times 10^{-1}\right), \mathrm{P}=0.783\right]$ (Figure $3 C$ ). The DWI analysis revealed no significant difference in the mean ADCs-measured at femoral heads-between the divers and controls (Table 4).

Five out of nine symptomatic divers were contacted after 5 years. Osteonecrosis was identified in the bilateral femoral heads of two divers with persistent hip pain, and the average water content in the femoral heads was nearly twice the initial value (initial: $7.25 \times 10^{-2} \mathrm{mM}$, follow-up: $15.0 \times 10^{-2} \mathrm{mM}$, mean difference: $0.077 \pm 0.13$ ). In contrast, no signal change in osteonecrosis was observed on the conventional MRI of the other three divers whose unilateral hip pain was relieved (initial: $6.64 \times 10^{-2} \mathrm{mM}$, follow-up: $6.83 \times 10^{-2} \mathrm{mM}$, mean difference: $0.003 \pm 0.01$ ).

\section{Discussion}

The present study revealed that dysbaric change in divers might be detected using quantitative MRS, which revealed increases in water content and UI of lipids, and a decrease in TLF in the femoral heads of divers with hip pain. The higher femoral head water content in symptomatic divers than in matched controls and asymptomatic divers (Table 4) suggests an association between hip pain in divers and increasing femoral head water content, concomitant with decrease in TLF. This result is consistent with the mechanism of early dysbaric ischaemia. According to this mechanism, blood passes from the arteries to the dilated and capacious venous sinusoids in bones, causing flow rates to drop and blood viscosity to increase and venous stasis, thrombosis, and finally, ischaemia to develop (4). The femoral head is mainly composed of cancellous bones, characterised by variable sized pores, comprising different relative percentages of water and fat. In cancellous bones, water molecules exist in three different forms: free water in pores, bound water in the collagen network (including at the collagen-mineral interface), and tightly bound water in the mineral phase (21). Free water is more prevalent in 
the boundary zones, whereas fat occupies primarily the central zone of each pore (22). No interaction between free water molecules and fat tissue exists owing to their separate locations in normal femoral heads, whereas water redistributes toward the central zone of pores and exhibits interactions with functional groups of fat tissue in the painful femoral heads of divers. Such changes may be related to intraosseous hypertension, which has a strong association with resting hip pain in venous ischaemia (23). Therefore, we supposed the hip pain in divers may indicate venous ischemia and intraosseous hypertension despite normal imaging studies.

Changes in UI reflect a dynamic relationship between the composition of fatty acids in the bone microenvironment and the metabolic requirements of cells (24). In this study, although statistical significance was not achieved, we noticed higher levels of unsaturated acids, with peaks at $2.03 \mathrm{ppm}$ (allyl methylene protons, L21), 2.77 ppm (divinyl methylene protons, L28) and $5.3 \mathrm{ppm}$ (olefinic protons, L53) on MRS, in symptomatic divers compared with controls (Table 4). Previous literature did not report the occurrence of increasing UI in the femoral heads of symptomatic divers, but did report its exclusive occurrence in osteoporotic femurs with fractures, where significantly decreased stearic acid content was concomitant with increased oleic acid content. In this circumstance, the decline in the level of saturated fatty acids resulted from increased supply of energetic substrate to marrow cells and osteoblasts for damage repair, whereas more unsaturated acids were produced to suppress type 2 cyclooxygenase expression in bone marrow and thereby excessive inflammation (25). As to early dysbaric ischemia, the increasing amount of unsaturated fatty acids might be related to their downregulation of excessive inflammation in bone marrow.

Dysbaric change is reversible in the femoral heads of symptomatic divers whose conventional imaging findings are normal. However, if the hip pain of workers persists owing to working in a hyperbaric environment, early dysbaric change can progress to frank osteonecrosis and increased water content in femoral heads. In this study, five out of nine symptomatic divers were contacted for follow-up MRS and DWI studies. Two divers with persistent hip pain continued to dive over these 5 years. Osteonecrosis was identified in the bilateral femoral heads, which also demonstrated an increase in their average water content. In contrast, no significant change in water content was noted in the other three divers, who had stopped diving, and their unilateral hip pain was relieved. Comparing these two groups of followers, the mean difference in water content in patients with osteonecrotic femoral heads was greater than that in patients with relieved symptoms and normal findings on MRI. Our result is consistent with a previous animal experiment, which proved that fluid shifts and any consequent changes in physiological parameters were transient and reversible at an early stage of gas-induced osmotic change within bones (26). In short, dysbaric changes in the femoral heads of symptomatic divers whose conventional imaging findings are normal are reversible, and an increased water content on MRS may indicate a risk for developing DON.

This study had some limitations. First, this is an exploratory study comparing two groups of divers. The significant findings in this article need to be validated by a more rigorous or cohort study. Second, a relatively small number of symptomatic divers with normal imaging findings of the hips were assessed. Usually, imaging findings are abnormal among divers with hip pain. Only a small fraction of symptomatic divers has normal findings on both plain radiography and conventional MRI. Third, our study was conducted in humans, rather than in animals, and therefore, bone ischaemia could not be manipulated. Moreover, because surgery was not prioritized for relieving hip pain in patients with normal imaging findings, we were unable to obtain a pathological diagnosis of bone ischaemia. As an alternative method, we assumed that hip pain was associated with reversible dysbaric change in the femoral heads of divers with normal imaging findings. Fourth, osteoporosis was visually excluded by two experienced musculoskeletal radiologists. Certain errors due to large differences in bone constitution between divers and controls could not be minimized because dual energy $\mathrm{X}$-ray absorptiometry was not performed to measure bone mineral density. Fifth, this was a cross-sectional study. The baseline ADC and quantitative MRS of subjects before diving were not obtained, and dysbaric change was defined by comparing divers and matched non-diver controls. In addition, only five out of nine symptomatic divers were contacted after 5 years, and the remaining patients were either lost to follow-up (in Groups 1 and 2) or unwilling to receive MRI scans owing to a lack of hip pain (in Groups 2). The absence of hip pain does not mean there is no dysbaric ischemia. Thus, a longitudinal cohort study is required to increase the accuracy of dysbaric change analysis in divers.

\section{Conclusions}

In conclusion, hip pain may be associated with reversible 
dysbaric changes in the femoral heads of divers with normal imaging findings. Quantitative MRS may be a feasible method for detecting dysbaric changes in the femoral head and potentially helping inform the risk for developing osteonecrosis.

\section{Acknowledgments}

Funding: This work was supported by a grant from the Medical Affairs Bureau Ministry of National Defense (MAB-105-058).

\section{Footnote}

Conflicts of Interest: All authors have completed the ICMJE uniform disclosure form (available at https://dx.doi. org/10.21037/qims-21-148). The authors have no conflicts of interest to declare.

Ethical Statement: The authors are accountable for all aspects of the work in ensuring that questions related to the accuracy or integrity of any part of the work are appropriately investigated and resolved. The study was conducted in accordance with the Declaration of Helsinki (as revised in 2013) and was approved by the Institutional Review Board of the Tri-Service General Hospital (TSGHIRB-2-103-05-078) and informed consent was taken from all individual participants before participation.

Open Access Statement: This is an Open Access article distributed in accordance with the Creative Commons Attribution-NonCommercial-NoDerivs 4.0 International License (CC BY-NC-ND 4.0), which permits the noncommercial replication and distribution of the article with the strict proviso that no changes or edits are made and the original work is properly cited (including links to both the formal publication through the relevant DOI and the license). See: https://creativecommons.org/licenses/by-nc-nd/4.0/.

\section{References}

1. Sharareh B, Schwarzkopf R. Dysbaric osteonecrosis: a literature review of pathophysiology, clinical presentation, and management. Clin J Sport Med 2015;25:153-61.

2. Gempp E, Louge P, de Maistre S. Predictive factors of dysbaric osteonecrosis following musculoskeletal decompression sickness in recreational SCUBA divers. Joint Bone Spine 2016;83:357-8.
3. Miyanishi K, Kamo Y, Ihara H, Naka T, Hirakawa M, Sugioka Y. Risk factors for dysbaric osteonecrosis. Rheumatology 2006;45:855-8.

4. Hutter CDD. Dysbaric osteonecrosis: a reassessment and hypothesis. Medical Hypotheses 2000;54:585-90.

5. Uguen M, Pougnet R, Uguen A, Loddé B, Dewitte JD. Dysbaric osteonecrosis among professional divers: a literature review. Undersea Hyperb Med 2014;41:579-87.

6. Bolte H, Koch A, Tetzlaff K, Bettinghausen E, Heller M, Reuter M. Detection of dysbaric osteonecrosis in military divers using magnetic resonance imaging. Eur Radiol 2005; 15:368-75.

7. Menezes NM, Connolly SA, Shapiro F, Olear EA, Jimenez RM, Zurakowski D, Jaramillo D. Early Ischemia in Growing Piglet Skeleton: MR Diffusion and Perfusion Imaging. Radiology 2007;242:129-36.

8. Hou CH, Shih TT, Liu CY, Li YD, Enright T. Proton MR spectroscopy of the femoral head-Evaluation of patients at risk for avascular necrosis. J Magn Reson Imaging 2006;24:409-17.

9. Mukisi MM, Bashoun K, Burny F. Sickle-cell hip necrosis and intraosseous pressure. Orthop Traumatol Surg Res 2009;95:134-8.

10. Takahashi T, Lapham G, Chavez LJ, Lee AK, Williams EC, Richards JE, Greenberg D, Rubinsky A, Berger D, Hawkins EJ, Merrill JO, Bradley KA. Comparison of DSM-IV and DSM-5 criteria for alcohol use disorders in VA primary care patients with frequent heavy drinking enrolled in a trial. Addict Sci Clin Pract 2017;12:17.

11. Tsunenari T, Tsutsumi M, Ohno K, Yamamoto $Y$, Kawakatsu M, Shimogaki K, Negishi H, Sugimoto T, Fukase M, Fujita T. Age- and gender-related changes in body composition in Japanese subjects. J Bone Miner Res 1993;8:397-402.

12. Lee EY, Kim D, Kim KM, Kim KJ, Choi HS, Rhee Y, Lim S-K. Age-Related Bone Mineral Density Patterns in Koreans (KNHANES IV). J Clin Endocrinol Metab 2012;97:3310-8.

13. Collaborative Osteonecrosis Group. Symptomatic Multifocal Osteonecrosis: A Multicenter Study. Clin Orthop Relat Res 1999;369:312-26.

14. Bammer R. Basic principles of diffusion-weighted imaging. Eur J Radiol 2003;45:169-84.

15. Ren J, Dimitrov I, Sherry AD, Malloy CR. Composition of adipose tissue and marrow fat in humans by $1 \mathrm{H}$ NMR at 7 Tesla. J Lipid Res 2008;49:2055-62.

16. Provencher SW. Automatic quantitation of localized in vivo $1 \mathrm{H}$ spectra with LCModel. NMR Biomed 
2001;14:260-4.

17. Di Pietro G, Capuani S, Manenti G, Vinicola V, Fusco A, Baldi J, Scimeca M, Hagberg G, Bozzali M, Simonetti G, Tarantino U. Bone Marrow Lipid Profiles from Peripheral Skeleton as Potential Biomarkers for Osteoporosis: A 1HMR Spectroscopy Study. Acad Radiol 2016;23:273-83.

18. Miyake Y, Yokomizo K, Matsuzaki N. Determination of unsaturated fatty acid composition by high-resolution nuclear magnetic resonance spectroscopy. J Am Oil Chem Soc 1998;75:1091-4.

19. Guillén MD, Ruiz A. 1H nuclear magnetic resonance as a fast tool for determining the composition of acyl chains in acylglycerol mixtures. Eur J Lipid Sci Technol 2003;105:502-7.

20. Guillén MD, Ruiz A. Rapid simultaneous determination by proton NMR of unsaturation and composition of acyl groups in vegetable oils. Eur J Lipid Sci Technol 2003;105:688-96.

21. Marinozzi F, Bini F, Quintino A, Corcione M, Marinozzi A. Experimental Study of Diffusion Coefficients of Water through the Collagen: Apatite Porosity in

Cite this article as: Lin TT, Hu CC, Hsu YC, Wang CC, Chiang SW, Wang CY, Chang WC, Huang GS. Utility of magnetic resonance spectroscopy and diffusion-weighted imaging for detecting changes in the femoral head in divers with hip pain at risk for dysbaric osteonecrosis. Quant Imaging Med Surg 2022;12(1):43-52. doi: 10.21037/qims-21-148
Human Trabecular Bone Tissue. Biomed Res Int 2014;2014:796519.

22. Capuani S. Water diffusion in cancellous bone. Microporous Mesoporous Mater 2013;178:34-8.

23. Arnoldi CC, Linderholm H, Müssbichler H. Venous engorgement and intraosseous hypertension in osteoarthritis of the HIP. J Bone Joint Surg Br 1972;54:409-21.

24. Pino AM, Miranda M, Figueroa C, Rodríguez JP, Rosen CJ. Qualitative Aspects of Bone Marrow Adiposity in Osteoporosis. Front Endocrinol (Lausanne) 2016;7:139.

25. Miranda M, Pino AM, Fuenzalida K, Rosen CJ, Seitz G, Rodríguez JP. Characterization of Fatty Acid Composition in Bone Marrow Fluid From Postmenopausal Women: Modification After Hip Fracture. J Cell Biochem 2016;117:2370-6.

26. Hills BA. Some physiological aspects of dysbarism - induced osteonecrosis. In: Dysbarium-related Osteonecrosis, a Symposium. USA: National Institute for Occupational Safety and Health, 1974. 